Editorial

\title{
Precision Medicine for Personalized Cancer Therapy
}

\author{
Ada Hang-Heng Wong and Chu-Xia Deng ${ }^{\bowtie}$ \\ Faculty of Health Sciences, University of Macau, Macau. \\ $\triangle$ Corresponding author: cxdeng@umac.mo
}

() 2015 Ivyspring International Publisher. Reproduction is permitted for personal, noncommercial use, provided that the article is in whole, unmodified, and properly cited. See http://ivyspring.com/terms for terms and conditions.

Received: 2015.10.18; Accepted: 2015.11.01; Published: 2015.11.17

Precision medicine is defined as the tailoring of clinical strategies based on genomic, genetic, behavioral and environment background of individual patients. Statistics indicated that traditional clinical practices sometimes lead to poor health outcomes and a waste of medical resources. Medical waste due to unnecessary treatment or ineffective treatment amounted to 75 billion US dollars per year $(30 \%$ of healthcare expenditure) in the US [1].

On $21^{\text {st }}$ January 2015, US President Barack Obama called for a Precision Medicine Initiative that select cancer as one of its immediate targets [2]. In March 2015, China announced their Precision Medicine Initiative, which plans to invest a total of 60 billion Renminbi in search for prediction of hereditary diseases in newborns, investigations on antibiotics resistance, preventive measures development and personalized cancer therapy. The United Kingdom also announced a 100,000 Genomes Project involving 70 thousand participants. Hence, precision medicine took center stage in the world's healthcare goals today.

Among all countries' objectives, fighting cancer is common to all. GLOBOCAN statistics revealed an estimated 14.1 million newly diagnosed cancer cases (excluding non-melanoma skin cancer), 8.2 million deaths from cancer and 32.6 million people living with cancer (within 5 years of cancer diagnosis) in 2012 worldwide [3]. The global economic loss of premature death and disability due to cancer was 895 billion US dollars in 2008, accounting for $1.5 \%$ of the world's gross domestic product (GDP) [4]. Additionally, the national cost for cancer care in the US amounted to 157 billion US dollars in 2010 [5]. The war against cancer started in 1971 when US President Richard Nixon signed the National Cancer Act. Although Nixon's War on Cancer largely failed due to a propensity of reasons, it energized many discoveries, including the discovery of numerous markers for cancers.

Cancer is the uncontrolled proliferation of cells in an organism leading to aggressive recession of normal cells/tissue, consequently death. Incidence of different cancers correlates to geographical location, sex, living habits, as well as Human Development Index (HDI) that is related to economic development and public health standards. In the beginning, people thought retroviruses were cancer drivers, which was proven wrong and partly attributed to the failure of Nixon's War. In 1976, the discovery of src in transforming normal fibroblasts established the term "oncogene" and led to massive search for oncogenes and tumor suppressor genes. However, this also brought the notion that "cancer is simply the result of gene mutation", resulting in wide application of reductionist approach to find "unification by simplification" in cancer research field. Later, substantial research on cancer evolution and metastasis revived the notion that "cancer is complex" and brought about a renewed concept of "individuality of cancer" nowadays. Nevertheless, cancer biologists Hanahan and Weinburg summarized ten hallmarks of all cancers, namely: (1) sustainable proliferation; (2) evasion of growth suppressors; (3) avoidance of immune destruction; (4) replicative immortality; (5) tumor-promoting inflammation; (6) invasion and metastasis; (7) angiogenesis induction; (8) genome instability and mutation; (9) resistance of cell death; and (10) reprogramming of energy metabolism [6].

In parallel to the evolution of the concept of cancer etiology, cancer therapy also evolved over time [4]. Osteosarcoma observed in Egyptian fossils was the first cancer uncovered where "no treatment" was stated. Later, mastectomy emerged. Tumor resection 
remains the routine treatment strategy for cancer therapy till now. Hormone therapy was developed in the $1^{\text {th }}$ century while radiotherapy was developed at the beginning of the $20^{\text {th }}$ century. Chemotherapy then came by to treat metastatic cancers. However, due to ignorance of cancer cause in the past, chemotherapy mainly manifests "one fits all" approach. Consequently, side effects including nausea, stem cell damage, hair loss, etc, occur frequently. Drug responsiveness of patients also diverge, resulting in poor therapeutic outcomes. Alternatively, targeted cancer therapies, although quite successful initially, frequently fail due to development of drug resistance and adverse side effects. For example, cisplatin is effective for treatment of many cancers, but resistance often develops. Inhibitors against mutant EGFR work well in many cancer patients, but refractory cancers eventually emerge and patients die due to cancer relapse and metastasis. Adverse drug reactions are a significant cause of hospitalization and deaths worldwide [7].

Therefore, precision medicine involves a multitude of techniques and research forces like Epigenetics, Proteomics and Metabolomics, and research itself will generate new tools and technology, which will benefit our scientific society as a whole. Furthermore, precision medicine preludes the era of patient-informed therapy. Revelation of patient's genome raises ethical issues of acknowledgement rights in compromise to psychological stress. It also raises political and legal issues regarding liabilities to clinical decision making. According to estimates, the annual cost for cancer treatment for each patient aged 65 or above, on the average, ranges from 5 to 115 thousand US dollars for initial year after diagnosis and from 57 to 141 thousand US dollars for the last year of life from cancer death [8]. Thus, despite from clinician's advice, cost immensely affects a patient's decision for his/her treatment and consequently, outcome. Nonetheless, precision medicine, which employs multi-omics to aid prognosis and personalized treatment, presents unprecedented opportunities for patient-informed therapy and subsequently improves clinical practice $[9,10]$.

Beyond development in research and clinical practice, the Precision Medicine Initiative also encourages partnership between the academia and healthcare providers. Implementation will also foster collaboration with the industrial and commercial sector, for example, pharmaceutical companies and medical device manufacturers that provide indispensable products and services. Lateral economic expansion, specifically, medical travel, is complimentary. Hence, the Precision Medicine Initiative collectively nurtures and coheres different social sectors on the path to success.

In conclusion, the cancer precision medicine will leverage advances in biotechnologies, such as next generation sequencing, proteomics, transcriptome, epigenetics, pharmacology and bioinformatics, to identify precise causes for cancers and develop tailor-fit personalized therapies (Figure 1). As part of the Precision Medicine Initiative, this is an indispensable and near-term goal towards broader application on other diseases including diabetes, obesity, etc. Hence, the recent call for Precision Medicine Initiative rapidly went into spotlight throughout the world.

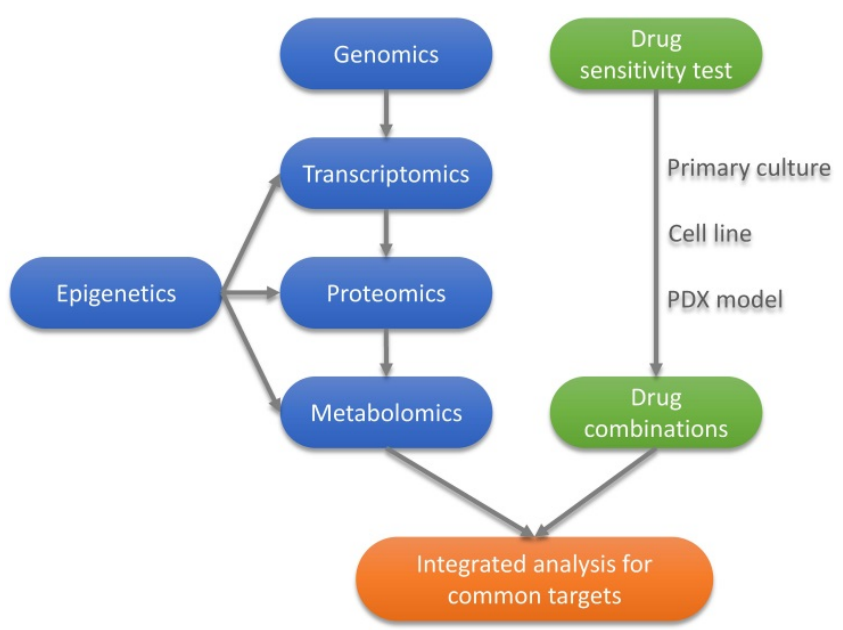

Figure 1. Precision Medicine for Personalized Cancer Therapy. Precision medicine involves a multitude of technologies in order to gain success. The combination of genomics analysis and drug screening will yield unpredicted pharmacological outcome and reveal information for precise disease diagnosis and treatment.

\section{Acknowledgments}

This work was supported by the startup funds and the Chair Professor Funds of the Faculty of Health Sciences, University of Macau, Macau SAR, China.

\section{Competing Interests}

The authors have declared that no competing interest exists.

\section{References}

[1] Lowrey A. Study of US Health Care System Finds Both Waste and Opportunity to Improve. The New York Times: Sep 11, 2012. (http://www.nytimes.com/2012/09/12/health/policy/waste-and-promise-s een-in-us-health-care-system.html?_r=0)

[2] [Internet] White House. The Precision Medicine Initiative. https://www.whitehouse gov/precision-medicine

[3] Stewart BW, Wild CP. World Cancer Report 2014. International Agency for Research on Cancer, World Health Organization. ISBN: 978-92-832-0429-9.

[4] [Internet] American Cancer Society. http://www.cancer.org/

[5] [Internet] National Cancer Institute. http://www.cancer.gov/

[6] Hanahan D, Weinberg RA. Hallmarks of cancer: the next generation. Cell, 2011, 144(5):646-674. 
[7] Davies EC, Green CF, Mottram DR, Pirmohamed M. Adverse drug reactions in hospitals: a narrative review. Curr Drug Saf. 2007, 2(1):79-87.

[8] Mariotto AB, Yabroff KR, et al. Projections of the cost of cancer care in the United States: 2010-2020. J Natl Cancer Inst, 2011, 103(2):117-128.

[9] Crystal AS, Shaw AT, et al. Patient-derived models of acquired resistance can identify effective drug combinations for cancer. Science, 2014, 346(6216):1480-1486

[10] Madhavan S, Gusev Y, et al. Genome-wide multi-omics profiling of colorectal cancer identifies immune determinants strongly associated with relapse. Front Genet, 2013, 4:236. 